\title{
Vacuolar Myopathies: Ultrastructural Studies Benefit Diagnosis
}

\author{
Ryan Goffredi ${ }^{1}$
}

${ }^{1}$ Children's Hospital Colorado, Aurora, United States

A case study of a 16-year-old male with symptoms of hypertrophic cardiomyopathy as well as skeletal muscle weakness is presented. A differential diagnosis is provided in light of specific laboratory testing and light microscopic findings which indicate a vacuolar myopathy affecting both cardiac and skeletal muscle. Vacuolar myopathies are a large and diverse group of disorders with a wide range of causes, clinical presentations, and ultrastructural findings. Select differential entities are then reviewed in terms of original discovery, pathophysiology, diagnostic testing, and ultrastructural findings. Following a discussion of etiologic and diagnostic information on each entity, the original case study is returned to and concluded based on the findings. The selected entities covered from the differential diagnosis are summarized below:

Glycogen Storage Disease Type II (Pompe Disease) This disease is caused by a mutation in the GAA gene which encodes the acid $\alpha$-glucosidase (formerly known as acid maltase) enzyme necessary for breaking down glycogen into glucose, within lysosomes, for cellular recycling [1]. A decrease in functional acid $\alpha$-glucosidase results in a build-up of lysosome-bound glycogen within various cell types in the body, including cardiac and skeletal muscle, and leads to cellular dysfunction. There are two ages of onset: infantile and "late" (which includes childhood, juvenile, and adult), of which, the infantile form is most severe and is life-threatening. By electron microscopy, myocytes are observed to contain large lysosomes filled with glycogen, and glycogen is seen spilling out into the cytoplasm and displacing the normal myofibrillar architecture. Autophagic vacuoles can be demonstrated in the cytoplasm of the myocytes, and the glycogen-filled lysosomes can also be identified in vascular endothelial cells and pericytes.

Danon disease results in hypertrophic cardiomyopathy and degenerative skeletal myopathy. Symptoms are more severe in males due to the X-chromosomal locus of the LAMP2 (lysosome-associated membrane protein 2) gene which is affected [3]. Under normal circumstances, the LAMP-2 protein is currently thought to be involved in many cell functions; primarily with fusion of the lysosomes to autophagosomes or directly with the plasma membrane itself. Therefore, a dysfunction in, or absence of, this protein results in the cell being unable to digest cellular materials, resulting in a build-up of material in these autophagosomes which may rupture and form large vacuoles that disrupt cell function. Ultrastructurally, vacuoles with aggregates of autophagosomal material and glycogen are seen within cardiac and skeletal muscle cells. Peculiar, membrane-bound vacuoles that are lined by a basal lamina can also be found in the myocyte cytoplasm, the pathologic significance of which is unknown.

XMEA is a slowly progressive, X-linked recessive myopathy of skeletal muscle only. It has only been found to affect males. The disease is caused by a mutation in the VMA21 gene which produces a chaperone protein required for the assembly of the V-ATPase complex. V-ATPase is a lysosomal proton pump that acidifies the interior of lysosomes. Malformation or absence of the V-ATPase pump results in non-acidification of lysosomes and therefore improper digestion of their contents, which is apparent also in autolysosomes [5]. This leads to lysosomes enlarged with undigested cellular material, including glycogen. Immunofluorescence microscopy shows a characteristic feature of this disease, which is the deposition of complement-associated membrane attack proteins over the myocyte sarcolemma. These 
proteins are also found within vacuoles in the myocyte cytoplasm. Ultrastructural studies demonstrate reduplication of the basement membrane surrounding the myocytes, and extrusion of autolysosomal material into the extracellular spaces. As in Danon disease, vacuoles lined by basement membrane can be observed within the myocyte cytoplasm.

Chloroquine is a drug first used as an antimalarial, and later found to be effective also in treating rheumatoid arthritis, systemic lupus erythematosis, and other diseases [6]. Its method of action is to raise intralysosomal $\mathrm{pH}$ and thereby inhibit lysosomal enzymes [7]. Prolonged use can induce a vacuolar myopathy that affects either cardiac or skeletal muscle, or both. The decreased activity of lysosomal enzymes leads to diminished digestion of effete materials, which results in enlarged lysosomes that can eventually rupture and cause cell damage. Ultrastructural findings include a marked increase in autolysosomal materials, cell damage, and curvilinear inclusions not found in the other entities described in this presentation. As with Pompe disease, lysosomal inclusions may be observed in vascular endothelial cells and pericytes.

References:

[1] A Nascimbeni et al, Cell Death and Differentiation 19 (2012), p. 1698.

[2] N Tachi et al, Pediatric Neurology 5 (1989), p. 60.

[3] I Nishino et al, Nature 406 (2000), p. 906.

[4] D Boucek, J Jirikowic and M Taylor, Genetics in Medicine 13 (2011), p. 563.

[5] N Ramachandran et al, Acta Neuropathologica 125 (2013), p. 439.

[6] M Eadie and T Ferrier, Journal of Neurology, Neurosurgery, and Psychiatry 29 (1966), p. 331.

[7] J Zirin, J Nieuwenhuis and N Perrimon, PLOS Biology 11 (2013), p. 1.

[8] I Nishino, Current Science 3 (2003), p. 64. 